\title{
Parámetros metabólicos, antioxidantes y competencia para el desarrollo embrionario de ovocitos bovinos madurados in vitro con L-Carnitina
}

\author{
Metabolic, antioxidant and competence parameters for the embryonic \\ development of bovine oocytes matured in vitro with l-carnitine
}

\author{
Natalia Jaramillo Bolívar ${ }^{1,3,4}$, Juan Miguel Arzuaga Cedeño ${ }^{2,3}$, John Jairo Giraldo \\ Giraldo ${ }^{1,3}$, Neil A. Vásquez Araque ${ }^{1,3}$
}

\section{Resumen}

Se evaluó el efecto antioxidante y metabólico de la L-Carnitina (L-C) durante la maduración in vitro de ovocitos bovinos sobre parámetros asociados a la calidad: cantidad relativa de lípidos, generación de especies reactivas de oxígeno (EROs), niveles de glutatión reducido (GSH), actividad mitocondrial y la competencia para el desarrollo embrionario posterior a la fertilización. Los complejos cúmulo ovocito fueron madurados por $24 \mathrm{~h}$ con y $\sin \mathrm{L}-\mathrm{C}(3.8 \mathrm{mM})$, e incubados con el fluoróforo específico para cada parámetro, Rojo Nilo (lípidos), Diclorofluoresceina diacetato (EROs), Monoclorobimane (GSH), Mitotracker Green (mitocondria). La intensidad de la fluorescencia fue analizada con el software ImageJ y normalizada al grupo control de ovocitos madurados sin LCarnitina. Para determinar la competencia del ovocito para el desarrollo embrionario, los ovocitos madurados fueron fertilizados y cultivados por 8 días. En los ovocitos madurados con L-C se encontró una disminución del $8.1 \%$ de la cantidad relativa de lípidos y en la generación de EROs del 41.6\%; mientras que la actividad mitocondrial aumentó en $160 \%$ con respecto al control, y mejoró la cinética y el porcentaje de blastocistos. Sin embargo, los niveles de GSH no se afectaron con la L-C. Los resultados soportan el efecto benéfico de la L-C durante la maduración in vitro del ovocito bovino para mejorar parámetros antioxidantes y metabólicos que se reflejan en la producción in vitro de embriones bovinos

Palabras clave: ovocito; maduración; L- carnitina; metabolismo, fluorocromo, $\beta$-oxidación

\footnotetext{
${ }^{1}$ Facultad de Ciencias, Universidad Nacional de Colombia Sede Medellín, Colombia

${ }^{2}$ Facultad Ciencias Agrarias, Universidad Nacional de Colombia Sede Medellín, Colombia

${ }^{3}$ Grupo de Investigación: Biotecnología Animal (BioA), Medellín, Colombia

${ }^{4}$ E-mail: njaramillob@unal.edu.co
}

Recibido: 7 de agosto de 2018

Aceptado para publicación: 5 de enero de 2019 
The antioxidant and metabolic effect of L-carnitine (LC) was evaluated during the in vitro maturation of bovine oocytes on parameters associated with quality: relative amount of lipids, generation of reactive oxygen species (ROS), reduced glutathione levels (GSH), mitochondrial activity and competence for embryonic development after fertilization. The cumulus-oocyte complexes were matured for $24 \mathrm{~h}$ with and without L-C (3.8 mM), and incubated with the specific fluorophore for each parameter, Nile Red (lipids), Dichlorofluorescein diacetate (EROs), Monoclorobimane (GSH), Mitotracker Green (mitochondria). The intensity of the fluorescence was analyzed with the ImageJ software and normalized to the control group of matured oocytes without L-Carnitine. To determine the competence of the oocyte for embryonic development, the matured oocytes were fertilized and cultured for 8 days. In oocytes matured with L-C a decrease of $8.1 \%$ of the relative amount of lipids and $41.6 \%$ in the generation of EROs was found, while mitochondrial activity increased by $160 \%$ with respect to control, plus improved the kinetics and percentage of blastocysts. However, GSH levels were not affected by L-C. The results support the beneficial effect of L-C during the in vitro maturation of the bovine oocyte to improve antioxidant and metabolic parameters that are reflected in the in vitro production of bovine embryos.

Key words: oocyte; maturation; L- carnitine; metabolism; fluorochrome; $\beta$-oxidation

\section{INTRODUCCIÓN}

Los sistemas de Producción in vitro de Embriones (PIVE) presentan dos grandes dificultades. La primera está asociada con los bajos porcentajes de blastocistos, indicador que ha conducido a la búsqueda de estrategias para mejorar la eficiencia y calidad de embriones bovinos. La segunda es la baja viabilidad y tasa de preñez de embriones sometidos a la criopreservación asociada a la acumulación de gotas lipídicas (Block et al., 2011). Teniendo en cuenta que uno de los pasos más críticos en la PIVE es la maduración del ovocito, la mejora de su calidad se ha convertido en un factor clave para aumentar la proporción y la calidad de blastocistos (Patrizio et al., 2007). Parámetros energéticos como los niveles de ATP (Cotterill et al., 2013) y actividad mitocondrial (Gottardi y Mingoti, 2009) están relacionados con una mayor competencia del ovocito para el desarrollo embrionario. Entre los moduladores del metabolismo más utilizados se encuentra el
Forskolin (Thomas et al., 2004), la insulina (Augustin et al., 2003) y la L-Carnitina (LC). Esta última es requerida durante la oxidación de los lípidos para el transporte de ácidos grasos desde el citosol al interior de las mitocondrias, para la generación de energía en forma de ATP. La L-C ejerce un importante efecto antioxidante, proporcionando múltiples mecanismos de protección celular al ovocito y al embrión en desarrollo (Abdelrazik y Agarwal, 2008).

La L-C, siendo un factor limitante para la activación e ingreso de los ácidos grasos a la matriz mitocondrial, se le ha evaluado en varios procesos biotecnológicos reproductivos, como la PIVE y la criopreservación (Held-Hoelker et al., 2017; Saraiva et al., 2018). Particularmente, se ha demostrado su importancia en la maduración nuclear del ovocito bovino, al utilizar Mildronate, un inhibidor de la biosíntesis de la L-C, presentando un efecto de dosis sobre la disminución del porcentaje de maduración del ovocito (Sanchez-Lazo et al., 2014). Por el contra- 
rio, la adición de L-C en la maduración in vitro (MIV) potencia o mejora el porcentaje de maduración nuclear (Somfai et al., 2011; Zare et al., 2015), inclusive del complejo cúmulo ovocito (CCO) de calidad intermedia (Knitlova et al., 2017).

La regulación del contenido de lípidos a través de su entrega directa a la mitocondria por la L-C es importante, puesto que garantiza el suministro de sustrato para la generación de energía y la disminución en la producción de gotas lipídicas en ovocitos inmaduros (Aon et al., 2014), además de mejorar la eficiencia en el desarrollo temprano de embriones bovinos (Jeong et al., 2009, Sutton-McDowall et al., 2012) y murinos (Dunning et al., 2010). Existe una relación entre la concentración del contenido de gotas lipídicas con la susceptibilidad a la criopreservación y la apoptosis de embriones bovinos producidos in vitro (Sudano et al., 2011). Los objetivos propuestos en este trabajo fueron evaluar el efecto del suplemento L-C durante la maduración in vitro de ovocitos bovinos sobre parámetros metabólicos (contenido lipídico, actividad mitocondrial), antioxidantes (niveles de producción de especies reactivas de oxígeno y de glutatión) y el porcentaje de desarrollo embrionario.

\section{Materiales y Métodos}

\section{Complejos Cúmulo Ovocito (CCOs)}

El material biológico para la obtención de los CCOs bovinos fue suministrado por una planta de faenado de Copacabana, Antioquia (Colombia). Se transportaron al laboratorio en solución tampón fosfato salino (PBS) estéril a $37^{\circ} \mathrm{C}$, donde se procedió a la aspiración de los folículos de diámetro 3-6 $\mathrm{mm}$ con agujas $\mathrm{N}^{\circ} 18$ y jeringa de $10 \mathrm{ml}$. El aspirado se colocó en tubos cónicos de $15 \mathrm{ml}$ a $37^{\circ} \mathrm{C}$. En caja de petri estéril de $60 \times 15$ $\mathrm{mm}$, bajo visión con estereomicroscopio, se seleccionaron los complejos CCOs de buena calidad, según criterios previamente establecidos (De Wit et al., 2000).

Los CCOs seleccionados fueron lavados con medio TCM-199 y transferidos a gotas de $50 \mu 1$ de medio de maduración (Gibco 12340-030) suplementado con $0.33 \mathrm{mM}$ de piruvato de sodio, $1 \mu \mathrm{g} / \mathrm{ml}$ de estradiol, $3 \%$ de suero fetal bovino SFB, $6 \mathrm{mg} / \mathrm{ml}$ de albúmina sérica bovina libre de ácidos grasos (BSA FAF, Sigma A6003), 1X de solución antibiótica, $50 \mu \mathrm{g} / \mathrm{ml}$ de gonadotropina coriónica humana (Chorulon ${ }^{\circledR}$ ), $1 \mu \mathrm{g} / \mathrm{ml}$ de FSH porcina (pFSHSigma F2293) y con o sin L-Carnitina a 3.8 $\mathrm{mM}$ (Phongnimitr et al., 2013) donde se incubaron por $24 \mathrm{~h}$ en grupos de 10-15. Las gotas fueron cubiertas con aceite mineral (Sigma M8410) e incubadas a $38.5^{\circ} \mathrm{C}, 5 \%$ $\mathrm{CO}_{2}$ en aire con $90 \%$ de humedad. Al finalizar el tiempo de maduración, se determinó el contenido lipídico, la actividad mitocondrial, los niveles de EROs y de GSH, y el desarrollo embrionario.

\section{Contenido Relativo de Lípidos}

Los CCOs fueron coloreados con Rojo Nilo (Sigma N3013, Molecular Probes) y los ovocitos madurados cultivados con o sin L$\mathrm{C}$, en grupos de 10 fueron incubados a $4{ }^{\circ} \mathrm{C}$ por $24 \mathrm{~h}$ en gotas de $250 \mu 1$ de medio de fijación que contiene Dulbecco's PBS, formaldehído (16\%) y glutaraldehído (50\%). Se lavaron en Dulbecco's PBS y pasaron a la solución del colorante $(1 \mu \mathrm{g} / \mathrm{ml})$ a temperatura ambiente por $24 \mathrm{~h}$. Luego se retiró el exceso de colorante lavando con Dulbecco's PBS y en grupos de 3 se pusieron en gotas de $10 \mu 1$ de Dulbecco's PBS sobre portaobjetos (Ballard; 2007), y se observaron a $200 \mathrm{X}$ en un microscopio de epifluorescencia (Nikon Eclipse 80i) con filtro C-FL G2A con una longitud de excitación a 515-560 nm y de emisión a $590 \mathrm{~nm}$. El área de ovocitos fue ajustada y las imágenes se guardaron en formato TIFF. La intensidad de fluorescencia de las gotas lipídicas en los ovocitos fue analizada por el software ImageJ v. 1.41 (National Institutes of Health, USA) y normalizadas con la medida de fluorescen- 
cia de los ovocitos sin tratamiento o control (Barceló-Fimbres y Seidel, 2011).

\section{Actividad Mitocondrial}

Los ovocitos madurados cultivados con o sin L-C fueron coloreados con Mito Tracker ${ }^{\circledR}$ green (Invitrogen M7514, Molecular Probes) y en grupos de 10 se incubaron a temperatura ambiente por $20 \mathrm{~min}$ en gotas de $50 \mu 1$ de Mito Tracker ${ }^{\circledR} 0.5 \mathrm{mM}$; luego se lavaron en Dulbecco's PBS y pasaron en grupos de 3 en gotas de $10 \mu 1$ de Dulbecco's PBS sobre portaobjetos (Ballard, 2007. Se observaron a $200 \mathrm{X}$ en un microscopio de epifluorescencia (Nikon Eclipse 80i) con filtro FITC/FLUO-3 (B-4A) con una longitud de emisión a $516 \mathrm{~nm}$ y de excitación a $490 \mathrm{~nm}$. La intensidad de fluorescencia en ovocitos fue analizada como se describió anteriormente.

\section{Glutatión Reducido (GSH)}

Los ovocitos madurados cultivados con o sin L-C fueron coloreados con el fluoróforo Monochlorobimane (mBCI) (Invitrogen M1381MP, Molecular Probes), y en grupos de 10 fueron incubados a temperatura ambiente por $15 \mathrm{~min}$ en gotas de $50 \mu \mathrm{lde} \mathrm{mBCI}$ $(0.25 \mathrm{mM})$. Luego se retiró el exceso de colorante lavando con Hepes y en grupos de tres se pusieron en gotas de $10 \mu 1$ de Hepes sobre portaobjetos (Ballard, 2007). Se observaron a $200 \mathrm{X}$ en un microscopio de epifluorescencia (Nikon Eclipse 80i) con filtro C-FL UV-2E/C con una longitud de excitación a $394 \mathrm{~nm}$ y de emisión a $490 \mathrm{~nm}$. La intensidad de fluorescencia en ovocitos fue analizada como se describió anteriormente.

\section{Especies Reactivas de Oxígeno (EROs)}

Los ovocitos madurados cultivados con o sin L-C fueron coloreados con el fluoróforo DicloroFluoresceína diacetato H2DCFDA (SIGMA-ALDRICH D6883, Molecular Probes) y en grupos de 10 se incubaron a temperatura ambiente por $20 \mathrm{~min}$ en gotas de $50 \mu 1$ de H2DCFDA $(10 \mu \mathrm{M})$. Luego se lavaron en Hepes (Ballard; 2007) y se observaron a $200 \mathrm{X}$ en un microscopio de epifluorescencia (Nikon Eclipse 80i) con filtro FITC/FLUO-3 (B-4A) con una longitud de excitación a $490 \mathrm{~nm}$ y de emisión a 514 $\mathrm{nm}$. La intensidad de fluorescencia en ovocitos fue analizada como se describió anteriormente.

\section{Fertilización de los Ovocitos y Cultivo de Presuntos Embriones}

Los CCOs maduros se transfirieron a gotas de $50 \mu 1$ de medio TL-FERT suplementado con cafeína $(1.4 \mathrm{mM}), 1 \mathrm{X}$ de solución antibiótica (ICN 1670049 MP Biomedicals) y se fertilizaron con semen descongelado perteneciente a un mismo lote de un solo donante, cuya viabilidad y calidad espermática se ajustó a los parámetros de calidad aplicados para bovinos, la concentración espermática final fue de $2 \times 10^{6}$ espermatozoides/ $\mathrm{ml}$. Las condiciones de fertilización fueron de $38.5{ }^{\circ} \mathrm{C}, 5 \%$ de $\mathrm{CO}_{2}$ y $90 \%$ de humedad relativa durante $18 \mathrm{~h}$ (Dode et al., 2002). A las $18 \mathrm{~h}$ de la incubación, los presuntos cigotos se desnudaron de las células del cúmulo y se cultivaron en gotas de $50 \mu 1$ de medio de cultivo KSOM-AA suplementado con $0.6 \mathrm{mg} / \mathrm{ml}$ de BSA-FAF, $1 \mathrm{X}$ de solución antibiótica y $3 \%$ de SBF por 8 días. Las condiciones de cultivo fueron $38.5{ }^{\circ} \mathrm{C}, 5 \%$ de $\mathrm{CO}_{2}$ y $90 \%$ de humedad relativa. La tasa de clivaje se determinó a las $48 \mathrm{~h}$ de cultivo sobre el total de ovocitos inseminados in vitro, y la tasa de blastocistos a los días 7 y 8 , sobre el total de ovocitos inseminados y de embriones en etapa de clivaje (Vásquez-Araque, 2014).

\section{Análisis Estadístico}

Se utilizó un diseño de bloques completo al azar y los supuestos de normalidad fueron evaluados por el test Kolmogorov-Smirnov (distribución de datos) y por la prueba de Brown-Forsythey (homogeneidad de varianzas). Posteriormente los datos fueron analizados por un análisis de varianza y las medias de los grupos fueron comparadas por el test de comparaciones múltiples Tukey 
(STATISTICA 10.0, StatSoft, Tulsa Oklahoma). Para todos los análisis fue considerado un valor $\mathrm{p}<0.05$.

\section{Resultados y Discusión}

La cantidad relativa de lípidos en el grupo de ovocitos madurados con L-C presentó un porcentaje promedio de intensidad de fluorescencia de $91.19 \%$, siendo significativamente menor $(\mathrm{p}<0.05)$ que el grupo madurado sin L-C (control) (100\%) (Figura 1a). Este efecto ha sido descrito en ovocitos bovinos con $2.5 \mathrm{mg} / \mathrm{ml}$ (Knitlova et al., 2017) y $3.03 \mathrm{mM}$ (Chankitisakul et al., 2013) de L-C, como también en blastocistos bovinos cultivados con $1.5 \mathrm{mM}$ de L-C (Ghanem et al., 2014). Esta respuesta de la L-C sobre las gotas lipídicas puede ser mediada en los ovocitos por la disminución en la expresión de los genes DGAT1, DGAT2 y PLIN2 (Perilipin 2) (Sanchez-Lazo et al., 2014) relacionados con la formación y expansión de las gotas lipídicas (Ghanem et al., 2014). Además, por la disminución del sustrato para la formación de triacilgliceroles, debido a que la L-C media el ingreso de los grupos acilos a la mitocondria para su oxidación (SanchezLazo et al., 2014), lo cual podría limitar la formación de las gotas lipídicas, conllevando a la disminución tanto de la biogénesis como de su expansión.

Al tener en cuenta que la L-C facilita el transporte de ácidos grasos al interior de la mitocondria, en donde pueden ser sustrato para la vía de la beta-oxidación, se determinó la actividad mitocondrial mediante la tinción con Mitotracker Green de los ovocitos madurados con L-C, los cuales presentaron un porcentaje promedio de intensidad $(260 \%)$; es decir, un aumento en la actividad mitocondrial de $160 \%$ con respecto al grupo control (100\%) (Figura 1B). Este aumento podría ser debido a la mayor disponibilidad de grupos acil-carnitina por la presencia de la L-C en el medio de maduración; sin embargo, otro factor que pudo haber influido en esta respuesta es un aumento en la expresión de CPT1 y CPT2, que aunque no se ha demostrado en ovocitos, se ha reportado en embriones cultivados en presencia de L-C (Ghanem et al., 2014) favoreciendo la beta oxidación y reflejándose con el aumento en la intensidad de fluorescencia (Dunning et al., 2010). Esta activación mitocondrial también se ha encontrado en ovocitos porcinos madurados con L-C a una concentración de 1.25 $\mathrm{mg} / \mathrm{ml}$ (Somfai et al., 2011) y en embriones bovinos cultivados con L-C a $1.5 \mathrm{mM}$ (Ghanem et al., 2014).

Otra propiedad atribuida a la L-C es su función antioxidante (Gülçin, 2006). Por lo tanto, se evaluaron parámetros antioxidantes, tales como los niveles de especies reactivas de oxígeno (EROs) y de glutatión reducido (GSH). Mediante la sonda fluorogénica H2DCFDA se encontró una disminución significativa $(\mathrm{p}<0.05)$ en la cantidad relativa de EROs al interior de los ovocitos madurados en presencia de L-C (58.3\%) (Figura 2), lo cual pudo deberse a las propiedades antioxidantes de esta molécula en sistemas acelulares (Gülçin, 2006) como en sistemas celulares (Somfai et al., 2011). Se ha demostrado que la suplementación con L-C reduce los niveles de EROs durante la maduración in vitro de ovocitos de ovinos (Mishra et al., 2016a), bovinos (Takahashi et al., 2013) у porcinos (Wu et al., 2011, You et al., 2012). Adicionalmente, se ha encontrado este efecto antioxidante sobre los niveles de EROs en embriones bovinos en el estadio de dos células, mas no así en etapas posteriores del desarrollo embrionario (Takahashi et al., 2013). Otros antioxidantes utilizados para disminuir las EROs, tanto en la maduración del ovocito como en el desarrollo embrionario, favoreciendo diferentes parámetros de eficiencia y calidad en la PIVE, son el ácido ascórbico (Vásquez-Araque, 2014), melatonina (Yang et al., 2017), $\alpha$-tocoferol (Li et al., 2014), quercetina y resveratrol (Sovernigo et al., 2017), cisteína y cisteamina intracelulares (Rocha-Frigoni et al., 2016, Sovernigo et al., 2017) y la catalasa extracelular (RochaFrigoni et al., 2016). 


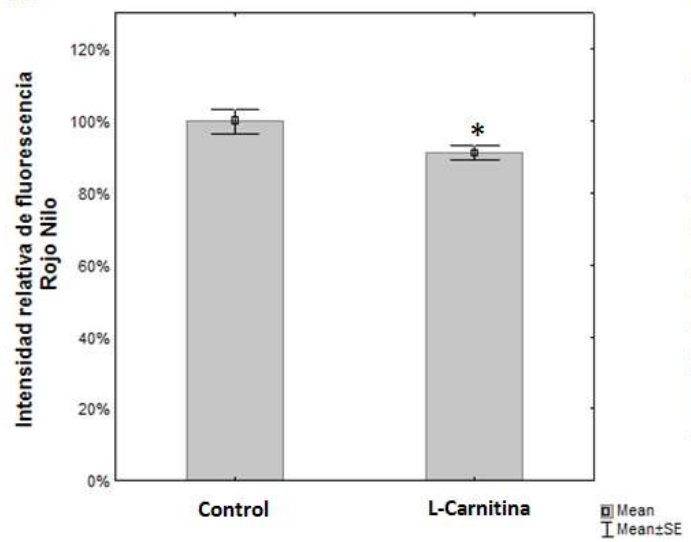

B.

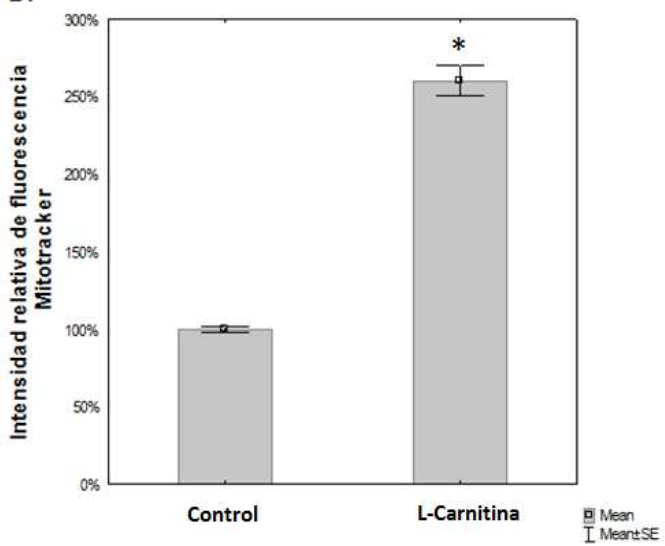

Figura 1. Ovocitos bovinos madurados con y sin L-Carnitina. A: Contenido relativo de lípidos (Tinción con Rojo Nilo). B. Actividad mitocondrial relativa (tinción con Mitotracker green). * Diferencia estadística significativa $(\mathrm{p}<0.05)$

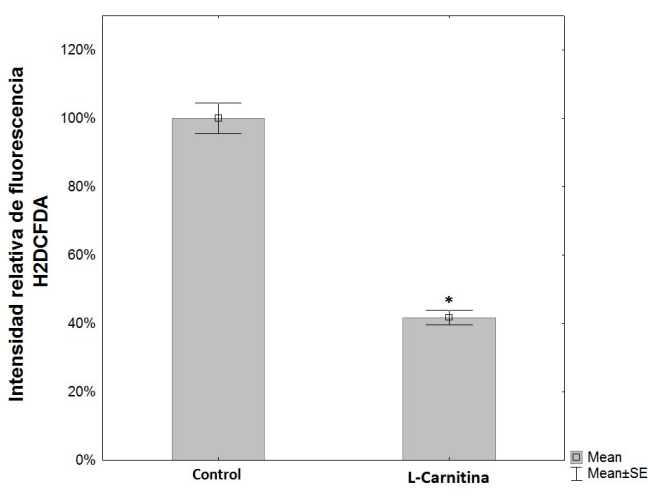

Figura 2. Producción relativa de especies reactivas de oxígeno (EROs) en ovocitos bovinos madurados con y sin L-Carnitina.

* Diferencia estadística significativa $(\mathrm{p}<0.05)$

La cantidad relativa de GSH, determinada mediante el fluoróforo Monochlorobimane, fue similar entre los ovocitos madurados con L-C $(95.37 \%)$ con respecto al control (100\%); sin embargo, se resalta que los niveles de GSH se mantuvieran a pesar del aumento en la actividad mitocondrial (160\%) y que los niveles de GSH dentro del ovocito no fueron afectados, debido a la presencia de glutatión reducido en la composición del medio de maduración (TCM-199, Gibco 12340-030). Otros suplementos con reconocida actividad antioxidante, tales como, quercetina, resveratrol y vitamina $C$, tampoco aumentaron los niveles de GSH en ovocitos de bovinos madurados in vitro (Sovernigo et al., 2017). No obstante, se ha reportado que la suplementación con L-C durante la maduración in vitro de ovocitos aumenta los niveles de GSH en ovinos (Mishra et al., 2016a), porcinos (Wu et al., 2011, You et al., 2012) y murinos (Zare et al., 2015).

Aunque los resultados de este trabajo no demostraron incremento en los niveles de GSH, este es considerado un marcador citoplásmico asociado con la calidad del ovocito, debido a que es el encargado de mantener el entorno redox al interior celular, además de ser esencial para la fertilización, lo cual se ha demostrado al inhibir su síntesis con buthionine sulphoximineal, bloqueando la formación del pronúcleo masculino luego de la fertilización (Sutovsky y Shatthen, 1997), mientras que al potenciar la producción de GSH suplementando con cisteamina (De Matos et al., 2002), cisteína, cistina y $\beta$ mercaptoetanol (De Matos y Furnus, 2000) se aumenta la tasa de blastocistos. 
Cuadro 1. Prueba de comparación de medias por el Test de Tukey para los parámetros de desarrollo (promedio \pm error estándar de la media)

\begin{tabular}{|c|c|c|c|c|}
\hline & & \multicolumn{2}{|c|}{ Ovocitos madurados } & \multirow{2}{*}{ Valor $\mathrm{p}$} \\
\hline & & Con L-Carnitina & Sin L-Carnitina & \\
\hline Clivaje (\%) & & $75.00(4.1)$ & $77.44(1.9)$ & 0.640 \\
\hline \multirow{2}{*}{$\begin{array}{l}\text { Porcentaje de blastocistos } \\
\text { con respecto a ovocitos } \\
\text { inseminados }\end{array}$} & Día 7 & $39.98(1.3)$ & $31.71(1.5)$ & 0.001 \\
\hline & Día 8 & $40.73(1.4)$ & $37.03(2.7)$ & 0.219 \\
\hline \multirow{2}{*}{$\begin{array}{l}\text { Porcentaje de blastocistos } \\
\text { con respecto a los clivajes }\end{array}$} & Día 7 & $54.15(2.3)$ & $41.85(1.1)$ & 0.001 \\
\hline & Día 8 & $55.08(2.3)$ & $49.32(3.0)$ & 0.147 \\
\hline
\end{tabular}

El porcentaje de blastocistos a partir de ovocitos inseminados in vitro al día 7 de cultivo fue mayor en el grupo de ovocitos madurados con L-C (39.98\%) que en el grupo control $(31.71 \%)$ (Cuadro 1). Los efectos benéficos de la maduración de ovocitos con L-C sobre el desarrollo embrionario han sido reportados en el bovino (Phongnimitr et al., 2013, Ghanem et al., 2014, Baldoceda et al., 2016), murino (Abdelrazik et al., 2009, Moawad et al., 2014, Zare et al., 2015), porcino (Somfai et al., 2011, Lowe et al., 2017, ) y ovinos (Mishra et al., 2016a,b); sin embargo, las concentraciones de L-C que presentaron mejor respuesta en el ovino fueron 7.5 $\mathrm{mM}$ y $10 \mathrm{mM}$ (Mishra et al., 2016a). Por otro lado, algunos estudios no encontraron efecto en la maduración de ovocitos bovinos con L-C sobre el porcentaje de blastocistos (Chankitisakul et al., 2013; McKeegan, 2015).

El porcentaje de embriones en etapa de clivaje que tienen la capacidad de llegar hasta la etapa de blastocisto (blastocisto/clivados) es un indicador de la calidad embrionaria (Lonergan et al., 2003, Vigneault et al., 2009). El porcentaje de blastocistos/clivados generados con ovocitos madurados con L-C (54.15\%) fue mayor que en el grupo control (41.85\%), lo que indica que la L-C puede mejorar la producción de embriones a través de mecanismos antioxidantes (You et al.,
2012; Mishra et al., 2016a), modulación del metabolismo (Moreira, 2015) y disminución del daño del ADN (Abdelrazik et al., 2009, Mishra et al., 2016b); además de favorecer las condiciones de cultivo, que permiten la expresión de genes implicados con el metabolismo lipídico (Sanchez-Lazo et al., 2014, Ghanem et al., 2014, Baldoceda et al., 2016, Saraiva et al., 2018), la replicación del ADN, factores de transcripción, quinasas (You et al., 2012), e implantación del embrión y mantenimiento de la preñez (Interferon Tau, IFNt) (Ghanem et al., 2014).

La producción de embriones (blastocistos) en el día 8 de cultivo fue similar entre los dos grupos (Cuadro 1); sin embargo, el grupo L-C n el día 7 presentó una mayor producción $(\mathrm{p}<0.005)$, lo cual indica que los embriones generados con ovocitos madurados con L-C tienen una cinética de desarrollo mayor que los embriones control. Aunque se controlaron varios factores que influyen en esta cinética, tales como el semen (del mismo individuo y eyaculado) (Alomar et al., 2008), suplementos energéticos y condiciones de cultivo (Lonergan et al., 2003), el sexo del embrión no fue controlado, ya que los embriones de sexo masculino tienen una cinética de desarrollo mayor (Alomar et al., 2008). 
Otros factores que modulan la expresión de genes que participan en la respuesta mediada por la L-C podrían tener un efecto sinérgico al inducido por la L-C para favorecer el desarrollo embrionario. Lo anterior es apoyado por la correlación entre los niveles de expresión de CPT2 y la competencia para el desarrollo embrionario (Gentile et al., 2004), como también la regulación por la LCarnitina de los niveles de CPT1 y CPT2 durante el desarrollo in vitro de embriones bovinos y murinos (Paczkowski et al., 2013; Ghanem et al., 2014; Baldoceda et al., 2016). Por otro lado, en el cerdo se ve reducida su expresión, lo que indica que la especie bovina es menos susceptible a perturbaciones en la oxidación de ácidos grasos (FAO, oxidation of fatty acids) (Paczkowski et al., 2013).

\section{Conclusiones}

- El suplemento del medio de maduración in vitro con L-Carnitina a una concentración de $3.8 \mathrm{mM}$ mejora la calidad del ovocito, según la disminución de la cantidad relativa de lípidos y de los niveles de Especies Reactivas de Oxígeno, el aumento de la actividad mitocondrial y el porcentaje de embriones en etapa de blastocisto.

- Se recomienda suplementar el medio de maduración con L-Carnitina para mejorar la eficiencia de biotecnologías como PIVE, clonación y criopreservación.

\section{Agradecimientos}

A la Gobernación del Cesar, CDT y el proyecto «Consolidación de las capacidades de ciencia, tecnología e innovación del sector agropecuario del departamento del Cesar». Al programa Joven Investigador del Departamento Administrativo de Ciencia, Tecnología e Innovación, Colciencias. Al Laboratorio de Genetica de la Universidad Nacional de Colombia (Sede Medellín).
Literatura Citada

1. Abdelrazik H, Agarwal A. 2008. Lcarnitine and assisted reproduction. Arch Med Sci 5: 43-47.

2. Abdelrazik H, Sharma R, Mahfouz R, Agarwal A. 2009. L-Carnitine decreases DNA damage and improves the in vitro blastocyst development rate in mouse embryos. Fertil Steril 91: 589596. doi: 10.1016/j.fertnstert.2007.11.067

3. Alomar M, Tasiaux H, Remacle $S$, George F, Paul D, Donnay I. 2008. Kinetics of fertilization and development, and sex ratio of bovine embryos produced using the semen of different bulls. Anim Reprod Sci 107: 48-61. doi: 10.1016/ j.anireprosci.2007.06.009

4. Aon M, Bhatt N, Cortassa S. 2014. Mitochondrial and cellular mechanisms for managing lipid excess. Front Physiol 5: 48-61. doi: 10.1016/j.anireprosci.2007.06.009

5. Augustin R, Pocar P, Wrenzycki C, Niemann H, Fischer B. 2003. Mitogenic and anti-apoptotic activity of insulin on bovine embryos produced in vitro. Reproduction 126: 91-99.

6. Baldoceda L, Gagné D, Ferreira CR, Robert C. 2016. Genetic influence on the reduction in bovine embryo lipid content by 1-carnitine. Reprod Fert Develop 28: 1172-1184. doi: 10.1071/ RD14215

7. Ballard C. 2007. Intracellular lipids in Bos indicus and Bos taurus oocytes. MSc thesis. USA: Louisiana State University. $146 \mathrm{p}$.

8. Barceló-Fimbres M, Seidel G. 2011. Effects of either glucose or fructose and metabolic regulators on bovine embryo development and lipid accumulation in vitro. Mol Reprod Dev 74: 1406-1418.

9. Block J, Hansen PJ, Loureiro B, Bonilla L. 2011. Improving posttransfer survival of bovine embryos produced in vitro: actions of insulin-like growth factor-1, colony stimulating fac- 
tor-2 and hyaluronan. Theriogenology 76 : 1602-1609. doi: 10.1016/j.theriogenology.2011.07.025

10. Chankitisakul V, Somfai T, Inaba Y, Techakumphu M, Nagai T. 2013. Supplementation of maturation medium with L-carnitine improves cryo-tolerance of bovine in vitro matured oocytes. Theriogenology 79: 590-598. doi: 10.1016/j.theriogenology.2012.11.011

11. Cotterill M, Harris SE, ColladoFernandez E, Lu J, Huntriss JD, Campbell BK, Picton HM. 2013. The activity and copy number of mitochondrial DNA in ovine oocytes throughout oogenesis in vivo and during oocyte maturation in vitro. Mol Hum Reprod 19: 444-450. doi: 10.1093/molehr/ gat013

12. De Matos DG, Furnus CC. 2000. The importance of having high glutathione (GSH) level after bovine in vitro maturation on embryo development effect of â-mercaptoethanol, cysteine and cysteine. Theriogenology 53:761-771. doi: 10.1016/S0093-691X(99)00278-2

13. De Matos D, Gasparrini B, Pasqualini S, Thompson J. 2002. Effect of glutathione synthesis stimulation during in vitro maturation of ovine oocytes on embryo development and intracellular peroxide content. Theriogenology 57: 1443-1451. doi: 10.1016/S0093691X(02)00643-XGet

14. De Wit A, Wurth Y, Kruip A, 2000. Effect of ovarian phase and follicle quality on morphology and developmental capacity of the bovine cumulus-oocyte complex. J Anim Sci 78: 1277-1283. doi: 10.1016/S0093-691X(02)00643-X

15. Dode MA, Rodovalho NC, Ueno VG, Fernandes CE. 2002. The effect of sperm preparation and co-incubation time on in vitro fertilization of Bos indicus oocytes. Anim Reprod Sci 69:1523. doi: 10.1016/S0378-4320(01)00148-8

16. Dunning K, Cashman K, Russell D, Thompson J, Norman R, Robker R. 2010. Beta-oxidation is essential for mouse oocyte developmental compe- tence and early embryo develop-ment. Biol Reprod 83: 909-918. doi: 10.1095/ biolreprod.110.084145

17. Gentile L, Monti M, Sebastiano V, Merico V, Nicolai $R$, Calvani $M$, Garagna $S$, et al. 2004. Single-cell quantitative RT-PCR analysis of Cpt $1 \mathrm{~b}$ and Cpt 2 gene expression in mouse antral oocytes and in preimplantation embryos. Cytogenet Genome Res 105: 215-221. doi: 10.1159/000078191

18. Ghanem N, Ha AN, Fakruzzaman M, Bang JI, Lee SC, Kong IK. 2014. Differential expression of selected candidate genes in bovine embryos produced in vitro and cultured with chemicals modulating lipid metabolism. Theriogenology 82: 238-250. doi: 10.1016/j.theriogenology.2014.03.024

19. Gottardi FP, Mingoti GZ. 2009. Maturação de oócitos bovinos e influência na aquisição da competência para o desenvolvimento do embrião. Rev Bras Reprod Anim 33: 82-94.

20. Gülçin I. 2006. Antioxidant and antiradical activities of L-carnitine. Life Sci 78: 803-811. doi: 10.1016/j.lfs.2005.05.103

21. Held-Hoelker E, Klein SL, Rings F, Salilew-Wondim D, Saeed-Zidane M, Neuhoff C, Tesfaye D, et al. 2017. Cryosurvival of in vitro produced bovine embryos supplemented with 1-carnitine and concurrent reduction of fatty acids. Theriogenology 96: 145-152. doi: 10.1016/j.theriogenology.2017.03.014

22. Jeong WJ, Cho SJ, Lee HS, Deb GK, Lee YS, Kwon TH, Kong IK. 2009. Effect of cytoplasmic lipid content on in vitro developmental efficiency of bovine IVP embryos. Theriogenology 72: 584589. doi: $10.1016 / \mathrm{j}$.theriogenology.2009.04.015

23. Knitlova D, Hulinska P, Jeseta M Hanzalova K1, Kempisty $B$, Machatkova M. 2017. Supplementation of 1-carnitine during in vitro maturation improves embryo development from less competent bovine oocytes. Theriogeno$\operatorname{logy} 102$ : 16-22. doi: $10.1016 / j$.theriogenology.2017.06.025 
24. Li Q, Wang YS, Wang LJ, Zhang H, Li RZ, Cui CC, Li WZ, Zhang Y, Jin YP. 2014. Vitamin C supplementation enhances compact morulae formation but reduces the hatching blastocyst rate of bovine somatic cell nuclear transfer embryos. Cell Reprogram 16: 290-297. doi: 10.1089/cell.2013.0088

25. Lonergan P, Rizos D, Gutierrez-Adan A, Fair T, Boland MP. 2003. Oocyte and embryo quality: effect of origin, cultura conditions and gene expression patterns. Reprod Domest Anim 38: 259267. doi: 10.1046/j.1439-0531.2003.00437.x

26. Lowe JL, Bartolac LK, Bathgate R, Grupen CG 2017. Supplementation of culture medium with L-carnitine improves the development and cryotolerance of in vitro-produced porcine embryos. Reprod Fert Develop 29: 23572366. doi: 10.1071/RD16442

27. McKeegan P. 2015. Metabolic regulation during early embryo development. PhD Thesis. UK: The University of Hull and the University of York. $278 \mathrm{p}$.

28. Mishra A, Reddy IJ, Gupta PS, Mondal S. 2016a. L-carnitine mediated reduction in oxidative stress and alteration in transcript level of antioxidant enzymes in sheep embryos produced in vitro. Reprod Domest Anim. doi: 10.1111/ rda.12682.

29. Mishra A, Reddy I, Gupta P, Mondal S. 2016b. Developmental regulation and modulation of apoptotic genes expression in sheep oocytes and embryos cultured in vitro with L-carnitine. Reprod Domest Anim 51:1020-1029. doi: 10.1111/ rda.12789

30. Moawad AR, Xu B, Tan SL, Taketo T. L-carnitine supplementation during vitrification of mouse germinal vesicle stage-oocytes and their subsequent in vitro maturation improves meiotic spindle configuration and mitochondrial distribution in metaphase II oocytes. Hum Reprod 29: 2256-2268. doi: 10.1093/ humrep/deu201
31. Moreira A. 2015. L-Carnitine and trans-10, cis-12 conjugated linoleic acid on in vitro bovine embryo production and cryopreservation. MSc thesis. Brasil: Univ Federal de Viscosia. $45 \mathrm{p}$.

32. Paczkowski M, Silva E, Schoolcraft WB, Krisher RL. 2013. Comparative importance of fatty acid beta-oxidation to nuclear maturation, gene expression, and glucose metabolism in mouse, bovine, and porcine cumulus oocyte complexes. Biol Reprod 88: 111. doi: 10.1095/biolreprod.113.108548

33. Patrizio P, Fragouli E, Bianchi V, Borini A, Wells D. Molecular methods for selection of the ideal oocyte. In: Reprod BioMed Online 15: 346-353.

34. Phongnimitr T, Liang Y, Srirattana K, Panyawai K, Sripunya N, Treetampinich C, Parnpai R. 2013. Effect of L-carnitine on maturation, cryo-tolerance and embryo developmental competence of bovine oocytes. Anim Sci J 84: 719725. doi: 10.1111 asj. 12067

35. Rocha-Frigoni NA, Leão BC, Dall'Acqua PC, Mingoti GZ. 2016. Improving the cytoplasmic maturation of bovine oocytes matured in vitro with intracellular and/or extracellular antioxidants is not associated with increased rates of embryo development. Theriogenology 86: 1897-1905. doi: 10.1016/j.theriogenology.2016.06.009

36. Sanchez-Lazo L, Brisard D, Elis S, Maillard V, Uzbekov R, Labas V, Desmarchais $A$, et al. 2014. Fatty acid synthesis and oxidation in cumulus cells support oocyte maturation in bovine. Mol Endocrinol 28: 1502-1521. doi: 10.1210/ me.2014-1049

37. Saraiva HFRA, Batista RITP, Alfradique VAP, Pinto PHN, Ribeiro LS, Oliveira CS, et al. 2018. L-carnitine supplementation during vitrification or warming of in vivo produced ovine embryos does not affect embryonic survival rates but alters CrAT and PRDX1 expression. Theriogenology 105: 150-157. doi: $10.1016 / j$.theriogenology.2017.09.022 
38. Somfai T, Kaneda M, Akagi S, Watanabe S, Haraguchi S, Mizutani E, Dang-Nguyen TQ, et al. 2011. Enhancement of lipid metabolism with Lcarnitine during in vitro maturation improves nuclear maturation and cleavage ability of follicular porcine oocytes. Reprod Fert Develop 23: 912920. doi: 10.1071/RD10339

39. Sovernigo TC, Adona PR, Monzani PS, Guemra S, Barros F, Lopes FG, Leal C. 2017. Effects of supplementation of medium with different antioxidants during in vitro maturation of bovine oocytes on subsequent embryo production. Reprod Domest Anim 52: 561-569. doi: 10.1111/ rda.12946

40. Sudano MJ, Paschoal DM, Rascado $S$, Magalhães LC, Crocomo LF, de Lima-Neto JF, Landim-Alvarenga $C$. 2011. Lipid content and apoptosis of in vitro-produced bovine embryos as determinants of susceptibility to vitrification. Theriogenology 75: 12111220. doi: 10.1016/j.theriogenology.2010.11 .033

41. Sutovsky P, Schatten G. 1997. Depletion of glutathione during bovine oocyte maturation reversibly blocks the decondensation of the male pronucleus and pronuclear apposition during fertilization. Biol Reprod 56: 1503-1512.

42. Sutton-McDowall ML, Feil D, Robker RL, Thompson JG, Dunning KR. 2012. Utilization of endogenous fatty acid stores for energy production in bovine preimplantation embryos. Theriogenology 77: 1632-1641. doi: 10.1016/ j.theriogenology.2011.12.008

43. Takahashi T, Inaba Y, Somfai T, Kaneda M, Geshi M, Nagai T, Manabe N. 2013. Supplementation of culture medium with L-carnitine improves development and cryotolerance of bovine embryos produced in vitro. Reprod Fert Develop 25: 589-599. doi: 10.1071/RD11262

44. Thomas RE, Armstrong DY, Gilchrist RB. 2004. Bovine cumulus cell-oocyte gap junctional communication during in vitro maturation in response to manipulation of cell-specific cyclic adenosine 3.5-monophosophate levels. Biol Reprod 70: 548-556. doi: 10.1095/ biolreprod.103.021204

45. Vásquez-Araque N. 2014. Efecto del suplemento insulina-transferrina-selenio (ITS) sobre la tasa de desarrollo y calidad de embriones bovinos producidos in vitro. En; Memorias II Congreso Internacional de Medicina Veterinaria y Zootecnia. Santa Marta, Colombia.

46. Vigneault C, McGraw S, Sirard MA. 2009. Spatiotemporal expression of transcriptional regulators in concert with the maternal to embryonic transition during bovine in vitro embryogenesis. Reproduction 137: 13-21. doi: 10.1530/ REP-08-0077

47. Wu GQ, Jia BY, Li JJ, Fu XW, Zhou GB, Hou YP, Zhu SE. 2011. L-carnitine enhances oocyte maturation and development of parthenogenetic embryos in pigs. Theriogenology 76: 785-793. doi: 10.1016/j.theriogenology.2011.04.011

48. Yang M, Tao J, Chai M, Wu H, Wang J, Li G, He C, Xie L, Ji P, Dai Y, Yang L, Liu G. 2017. Melatonin improves the quality of inferior bovine oocytes and promoted their subsequent IVF embryo development: mechanisms and results. Molecules 27: 22. doi: 10.3390/ molecules22122059

49. You J, Lee J, Hyun SH, Lee E. 2012. L-carnitine treatment during oocyte maturation improves in vitro development of cloned pig embryos by influencing intracellular glutathione synthesis and embryonic gene expression. Theriogenology 78: 235-243. doi: 10.1016/ j.theriogenology.2012.02.027

50. Zare Z, Farahani R, Salehi M, Piryaei A, Novin G, Fathabadi F, Mohammadi M, Dehghani-Mohammadabadi $M$. 2015. Effect of L-carnitine supplementation on maturation and early embryo development of immature mouse oocytes selected by brilliant cresyle blue staining. J Assist Reprod Gen 32: 635-643. doi: 10.1007/s10815-015-0430-5 